JAN PALCZEWSKI (Warszawa)

\title{
ARBITRAGE AND PRICING IN A GENERAL MODEL WITH FLOWS
}

Abstract. We study a fundamental issue in the theory of modeling of financial markets. We consider a model where any investment opportunity is described by its cash flows. We allow for a finite number of transactions in a finite time horizon. Each transaction is held at a random moment. This places our model closer to the real world situation than discrete-time or continuous-time models. Moreover, our model creates a general framework to consider markets with different types of imperfection: proportional transaction costs, frictions on the numeraire, etc.

We develop an analog of the fundamental theorem of asset pricing. We show that lack of arbitrage is essentially equivalent to existence of a Lipschitz continuous discount process such that the expected value of discounted cash flows of any investment is non-positive. We address the question of contingent claim pricing and hedging.

1. Introduction. Financial mathematics is mostly engaged in design of models of the financial world. In addition to being "good copies" of reality these models should be tractable by mathematics. There are several reasons to develop models. We will mention two of them: better understanding of the "nature of the market" and pricing of contingent claims. A key notion for these questions is lack of arbitrage. It has always had an economic meaning of inability to acquire positive gains without any risk. However, incorporating it in the models of the market required major refinements. Simple discrete-time models used the most intuitive definition: a self-financing strategy with zero initial capital is an arbitrage opportunity if it produces a non-negative final value with probability one and positive final value with probability greater than zero (Bingham-Kiesel [1]). During its history financial mathematics developed a lot of theories that addressed

2000 Mathematics Subject Classification: 91B24, 91B28, 46 B99. 
different aspects of non-ideal market. These were lack of numeraire, transaction costs, short-sale constraints, partial knowledge, etc. But there has been a strong need for a general framework that would cover all those aspects and allow research into the "nature" of arbitrage and pricing.

A remarkable step in this direction was made by Jouini and Napp in [3]. They adopted a model where all investment opportunities were described by their cash flows. For instance, in such a model the investment which consists of buying, in a perfect financial model, at date $t_{1}$ one unit of a risky asset whose price process is given by $\left(S_{t}\right)_{t \in \mathbb{R}_{+}}$and selling this unit at date $t_{2}>t_{1}$, is described by a process $\left(\Phi_{t}\right)_{t \in \mathbb{R}_{+}}$which is null outside $\left\{t_{1}, t_{2}\right\}$ and which satisfies $\Phi_{t_{1}}=-S_{t_{1}}, \Phi_{t_{2}}=S_{t_{2}}$. Each investment $\left(\Phi_{t}\right)_{t \in \mathbb{R}_{+}}$is null everywhere but at a finite number of points. The market is defined as a positive convex cone of such investments.

The framework proposed by Jouini and Napp is quite general. It allows for a wide class of models: with perfect numeraire, with different rates of borrowing and lending, with convex cone constraints on the quantities of assets held by investors (e.g. short-sale constraints) and with proportional transaction costs. Moreover, it is much closer to the real world than classical discrete-time models. It generalizes the notion of discrete time. Each investment consists of a finite number of cash flows at deterministic moments, but different investments can have cash flows at different moments. It reflects the mechanism of trading in the real world. No investor can make an infinite number of transactions in a finite time. However, one can argue that it is possible to make transactions at a finite number of stopping times, for instance when the price of the stock reaches a certain limit. A small progress in this direction was made by Napp in [5]. She modified the model in such a way that one could consider stopping times with a countable number of values. In both papers the analog of the Fundamental Theorem of Asset Pricing was presented. It stated that there is no possibility of free lunch if and only if there exists an adapted discount process $g_{t}$ with a uniformly bounded and strictly positive modification such that the expected value of discounted cash flows of any investment is non-positive.

In the present paper we make further generalizations of Jouini's approach. We construct a model that goes along with the following real world rules:

- at each moment an agent has full knowledge of what has happened up to that time,

- an agent can make only a finite number of transactions in a finite time horizon,

- each transaction consists of a random cash flow occurring at a random moment; the decision of choosing the moment is made according to the knowledge available to the agent. 
We satisfy these rules by allowing for random cash flows at any bounded stopping time. Therefore we exploit advantages of discrete-time and continuous-time models. Continuous models express our perception of time, but continuous trading is a huge simplification to what one can observe. On the contrary, discrete-time models go along our requirements for trading (there must be a gap between subsequent transactions). However, it allows for transactions only at a finite set of moments in a finite horizon.

For the constructed model we derive an analog of the Fundamental Theorem of Asset Pricing in the spirit of Jouini and Napp ([3]). We prove that the absence of free lunch (a stronger version of the no-arbitrage condition) is equivalent to the existence of a discount process $g_{t}$ such that the expected value of discounted cash flows of any investment is non-positive. The process $g_{t}$ has uniformly bounded and Lipschitz continuous trajectories, a natural property from the economic point of view.

The last sections of the paper are devoted to contingent claim pricing. We show how the theorems from the previous sections can be used to obtain links between arbitrage pricing (in the spirit of Harrison and Kreps [2]) and hedging (a super-replication cost). We prove that the upper limit of the no-arbitrage interval of option prices is equal to the super-replication cost. A paper by Napp ([5]) provides a much wider coverage of the pricing issues in the model with flows, but with a different approach.

In Section 2 we define a model of the market. The next two sections are devoted to designing a framework that enables us to define the notion of arbitrage and free lunch. In Section 3 we introduce a Banach space $\mathcal{M}$ and prove some of its properties. Section 4 is based on a seminar by Schwartz [7] and defines a space $L_{\mathbb{P}}^{1}(\Omega, \mathcal{M})$ of integrable functions and its dual. Section 5 provides a representation of investments as elements of $L_{\mathbb{P}}^{1}(\Omega, \mathcal{M})$ and defines conditions of no arbitrage and no free lunch. We prove a Fundamental Theorem of Asset Pricing, i.e. existence of discount processes (our proof is based on the proof by Jouini and Napp [3]). Contingent claim pricing and hedging are addressed in Section 6.

2. A model. The model presented in this paper is a generalization of the model proposed by Jouini and Napp in [3]. We are given a probability space $(\Omega, \mathcal{F}, \mathbb{P})$ representing all states of the world. The probability distribution $\mathbb{P}$ is the real world probability. The knowledge about the world is encoded in the filtration $\left(\mathcal{F}_{t}\right)_{t \in \mathbb{R}_{+}}$satisfying the usual conditions (completeness and right continuity) with $\mathcal{F}_{0}$ being trivial (we have perfect knowledge of the world at time zero). In this probabilistic framework we define investment opportunities. Each investment opportunity is described by its cash flows. Every cash flow occurs at a random moment defined by a bounded stopping 
time. A positive cash flow represents obtaining money, a negative flow means paying.

Definition 2.1. An investment opportunity $\left(\Phi_{t}\right)_{t \in \mathbb{R}_{+}}$is an $\left(\mathcal{F}_{t}\right)_{t \in \mathbb{R}_{+}}{ }^{-}$ adapted process that can be written in the form

$$
\Phi_{t}=\sum_{i=1}^{n} \Phi_{\tau_{i}} 1_{\tau_{i}}(t)
$$

for some $n>0$ and a sequence of bounded stopping times $\tau_{1}, \ldots, \tau_{n}$ such that $\Phi_{\tau_{i}} \in L^{1}\left(\Omega, \mathcal{F}_{\tau_{i}}, \mathbb{P}\right)$ for every $i \in\{1, \ldots, n\}$. We denote by $\Delta$ the set of all investments.

The market is defined by available investment opportunities. We assume that each investment is infinitely divisible, i.e. for any investment $\left(\Phi_{t}\right)_{t \in \mathbb{R}_{+}}$ there exists an investment $\left(\lambda \Phi_{t}\right)_{t \in \mathbb{R}_{+}}$for any non-negative $\lambda$. Moreover, if there are two different investment opportunities $\left(\Phi_{t}\right)_{t \in \mathbb{R}_{+}},\left(\Psi_{t}\right)_{t \in \mathbb{R}_{+}}$then an agent can submit to both of them at the same time, so there exists an investment that is the sum of $\left(\Phi_{t}\right)_{t \in \mathbb{R}_{+}}$and $\left(\Psi_{t}\right)_{t \in \mathbb{R}_{+}}$.

Definition 2.2. A market $J$ is a positive convex cone of investment opportunities, i.e.

1) $\Phi_{1}, \Phi_{2} \in J \Rightarrow \Phi_{1}+\Phi_{2} \in J$

2) $\Phi \in J, a \in \mathbb{R}_{+} \Rightarrow a \Phi \in J$.

EXAMPLE 2.1. We will construct a cone $J$ representing a perfect market with $N$ assets whose price processes are given by $\left(S_{t}^{i}\right)_{t \in \mathbb{R}_{+}}, i=1, \ldots, N$. We require that for any $i=1, \ldots, N$ and $\tau$ a bounded stopping time, $S_{\tau}^{i} \in$ $L^{1}\left(\Omega, \mathcal{F}_{\tau}, \mathbb{P}\right)$. We assume that there are no short-sale constraints or any other frictions. Define

$$
\Phi_{t}^{\Theta, \tau, \sigma, S}=\Theta(t)\left(1_{\tau}(t) S_{\tau}-1_{\sigma}(t) S_{\sigma}\right),
$$

where $\tau, \sigma$ are bounded stopping times and $\Theta \in L^{\infty}\left(\Omega, \mathcal{F}_{\tau \wedge \sigma}, \mathbb{P}\right)$. Denote by $J$ the positive convex cone generated by all investments of the form (1). The set $J$ defines a market.

Because of the generality of our model we cannot use the classical approach to define the notion of free lunch. In the following sections we develop a theory that enables us to formulate this definition.

3. A Banach space $\mathcal{M}$. The aim of this section is to design a Banach space $\mathcal{M}$ fulfilling a few crucial requirements. We list some of them:

- for every $t \in \mathbb{R}_{+}$there exists an element $\delta_{t} \in \mathcal{M}$,

- $\left\|\delta_{s}-\delta_{t}\right\|_{\mathcal{M}}>0$ for $s \neq t$

- the space $\mathcal{M}$ supports finite linear combinations of $\delta_{t}$, 
- it is possible to decide "easily" whether all coefficients in a linear combination of $\delta_{t}$ are positive,

- for $t_{n} \rightarrow t, a_{n} \rightarrow a$ we have $a_{n} \delta_{t_{n}} \rightarrow a \delta_{t}$.

We start with a definition of a normed linear space $M$. Let

$$
M=\left\{\mu: \mathbb{R}_{+} \rightarrow \mathbb{R}: \operatorname{supp} \mu \text { is finite }\right\} .
$$

It is easy to see that $M$ is actually a linear space. We shall equip it with a norm. First we denote by $\mathcal{D}$ the set of functions bounded by 1 and Lipschitz continuous with constant 1 , i.e.

$$
\mathcal{D}=\left\{f: \mathbb{R}_{+} \rightarrow \mathbb{R}: \forall t, s \in \mathbb{R}_{+},|f(t)| \leq 1,|f(t)-f(s)| \leq|t-s|\right\} .
$$

Then we define a functional on $M$ by

$$
\|\mu\|_{M}=\sup \left\{\left|\sum_{t \in \mathbb{R}_{+}} f(t) \mu(t)\right|: f \in \mathcal{D}\right\} .
$$

Lemma 3.1. $\left(M,\|\cdot\|_{M}\right)$ is a normed linear space.

Proof. First we show that $\|\cdot\|_{M}$ is well defined for all elements of $M$. Fix $\mu \in M$. Then clearly $\sum_{t \in \mathbb{R}_{+}}|\mu(t)|<\infty$ and $\|\mu\|_{M} \leq \sum_{t \in \mathbb{R}_{+}}|\mu(t)|$.

We shall show that $\|\cdot\|_{M}$ is a norm:

(1) $\|\mu\|_{M}=0$ iff $\mu=0$,

(2) $\|\lambda \mu\|_{M}=|\lambda|\|\mu\|_{M}$,

(3) $\|\mu+\nu\|_{M} \leq\|\mu\|_{M}+\|\nu\|_{M}$.

In (1), one implication is clear. To show the other let $\mu \neq 0$. Thus $\mu$ has a representation $\mu=\sum_{k=1}^{K} \alpha_{k} \delta_{t_{k}}$ for some $K,\left(\alpha_{k}\right) \subset \mathbb{R} \backslash\{0\}, 0 \leq t_{1}<\ldots$ $<t_{K}$ (we denote by $\delta_{t}(s)$ the function $1_{t-s}$ ). Put

$$
f(t)= \begin{cases}\left(t_{2}-t_{1}\right) \wedge 1, & t \leq t_{1}, \\ \left(t_{2}-t\right) \wedge 1, & t_{1}<t \leq t_{2}, \\ 0, & t>t_{2},\end{cases}
$$

where $a \wedge b=\min (a, b)$. Then $f \in \mathcal{D}$ and $\left|\sum_{t \in \mathbb{R}_{+}} f(t) \mu(t)\right|=\left|\alpha_{1}\right|\left[\left(t_{2}-t_{1}\right) \wedge 1\right]$. Thus $\|\mu\|_{M} \geq\left|\alpha_{1}\right|\left[\left(t_{2}-t_{1}\right) \wedge 1\right]>0$. Condition (2) results from the fact that $\left|\sum_{t \in \mathbb{R}_{+}} f(t) \lambda \mu(t)\right|=|\lambda|\left|\sum_{t \in \mathbb{R}_{+}} f(t) \mu(t)\right|$.

The proof of $(3)$ is also simple. Fix $f \in \mathcal{D}$. Then

$$
\begin{aligned}
\left|\sum_{t \in \mathbb{R}_{+}} f(t)(\mu(t)+\nu(t))\right| & \leq\left|\sum_{t \in \mathbb{R}_{+}} f(t) \mu(t)\right|+\left|\sum_{t \in \mathbb{R}_{+}} f(t) \nu(t)\right| \\
& \leq \sup _{h \in \mathcal{D}}\left|\sum_{t \in \mathbb{R}_{+}} h(t) \mu(t)\right|+\sup _{h \in \mathcal{D}}\left|\sum_{t \in \mathbb{R}_{+}} h(t) \nu(t)\right| \\
& =\|\mu\|_{M}+\|\nu\|_{M} \text {. }
\end{aligned}
$$


Once we have defined the space $M$ we shall calculate the norms of some simple elements. We denote by $\delta_{t}$ the function in $M$ which is null at all points but $t$ and equals 1 at $t$, so $\delta_{t}(s)=1_{t=s}$.

Lemma 3.2. Let $\alpha, \beta \geq 0, t, s \in \mathbb{R}_{+}, t \neq s$. Then

(1) $\left\|\alpha \delta_{t}+\beta \delta_{s}\right\|_{M}=\alpha+\beta$,

(2) $\left\|\alpha \delta_{t}-\beta \delta_{s}\right\|_{M}= \begin{cases}|\alpha-\beta|+|t-s|(\alpha \wedge \beta), & |t-s| \leq 2, \\ \alpha+\beta, & |t-s|>2 .\end{cases}$

Proof. Part (1) is obvious by taking $f=1 \in \mathcal{D}$. Part (2) is more complicated. Set $\varepsilon=|t-s|$. If $\varepsilon>2$ then there exists $f \in \mathcal{D}$ such that $f(t)=1$ and $f(s)=-1$. This function realizes the supremum in the definition of the norm and yields $\left\|\alpha \delta_{t}-\beta \delta_{s}\right\|_{M}=\alpha+\beta$.

Let $\varepsilon \leq 2$. Assume that $\alpha \geq \beta$. We can give an equivalent characterization of the norm $\left\|\alpha \delta_{t}-\beta \delta_{s}\right\|_{M}$. We are only interested in possible values of functions in $\mathcal{D}$ at the points $s, t$. Set $\widetilde{\mathcal{D}}=\{(a, b):|a| \leq 1,|b| \leq 1,|a-b| \leq \varepsilon\}$. Then $\left\|\alpha \delta_{t}-\beta \delta_{s}\right\|_{M}=\sup _{(a, b) \in \widetilde{\mathcal{D}}}|a \alpha-b \beta|$. The function $b \mapsto a_{0} \alpha-b \beta$ is decreasing, so

$$
\begin{aligned}
F\left(a_{0}\right) & :=\sup _{\left(a_{0}, b\right) \in \widetilde{\mathcal{D}}}\left|a_{0} \alpha-b \beta\right| \\
& =\max \left(\left|a_{0} \alpha-\left[\left(a_{0}-\varepsilon\right) \vee-1\right] \beta\right|,\left|a_{0} \alpha-\left[\left(a_{0}+\varepsilon\right) \wedge 1\right] \beta\right|\right) .
\end{aligned}
$$

Hence $\left\|\alpha \delta_{t}-\beta \delta_{s}\right\|_{M}=\sup _{a \in[-1,1]} F(a)$. Simple calculations lead to the conclusion that $\left\|\alpha \delta_{t}-\beta \delta_{s}\right\|_{M}=\alpha-\beta+\varepsilon \beta$.

Having the above lemma we can see that $M$ is not complete. Consider the sequence $\mu_{n}=\sum_{i=1}^{n} 2^{-i} \delta_{2 i}$. It is a Cauchy sequence: $\left\|\mu_{n}-\mu_{m}\right\|_{M}=$ $\sum_{i=m}^{n} 2^{-i}$ for $n>m$. But it does not have a limit in $M$. A good candidate for the limit would be $\mu_{\infty}$, but it would need to have an infinite support.

Definition 3.3. Denote by $\left(\mathcal{M},\|\cdot\|_{\mathcal{M}}\right)$ the completion of $M$ with the norm generated by $\|\cdot\|_{M}$.

Now we shall study the space $\mathcal{M}^{\prime}$ of continuous linear functionals on $\mathcal{M}$. We will see that there exists a 1-1 correspondence between $\mathcal{M}^{\prime}$ and the set of all Lipschitz continuous bounded functions.

Lemma 3.4. Let $\mu^{*} \in \mathcal{M}^{\prime}$. The function $f(t)=\left\langle\mu^{*}, \delta_{t}\right\rangle$ is bounded and Lipschitz continuous, i.e. there exists a constant $C$ such that $|f(t)| \leq C$ and $|f(t)-f(s)| \leq C|t-s|$. In particular, we can take $C=\left\|\mu^{*}\right\|_{\mathcal{M}^{\prime}}$.

Proof. To prove the boundedness we exploit the continuity of $\mu^{*}$. For $t \in \mathbb{R}_{+}$,

$$
|f(t)|=\left|\left\langle\mu^{*}, \delta_{t}\right\rangle\right| \leq\left\|\mu^{*}\right\|_{\mathcal{M}^{\prime}}\left\|\delta_{t}\right\|_{\mathcal{M}}=\left\|\mu^{*}\right\|_{\mathcal{M}^{\prime}} .
$$


Now fix $t, s \in \mathbb{R}_{+}$. In a similar way we obtain

$$
|f(t)-f(s)|=\left|\left\langle\mu^{*}, \delta_{t}-\delta_{s}\right\rangle\right| \leq\left\|\mu^{*}\right\|_{\mathcal{M}^{\prime}}\left\|\delta_{t}-\delta_{s}\right\|_{\mathcal{M}} .
$$

From Lemma 3.2 we get $\left\|\delta_{t}-\delta_{s}\right\|_{\mathcal{M}}=|t-s| \wedge 2 \leq|t-s|$, which finishes the proof with $C=\left\|\mu^{*}\right\|_{\mathcal{M}^{\prime}}$.

Lemma 3.5. Let $f: \mathbb{R}_{+} \rightarrow \mathbb{R}$ be a bounded and Lipschitz continuous function, i.e. $|f(t)| \leq C$ and $|f(t)-f(s)| \leq C|t-s|$ for some constant $C$. Then there exists exactly one continuous linear functional $\mu^{*}$ on $\mathcal{M}$ such that $\left\langle\mu^{*}, \delta_{t}\right\rangle=f(t)$. Moreover, $\left\|\mu^{*}\right\|_{\mathcal{M}^{\prime}} \leq C$.

Proof. We can restrict ourselves to the case $C=1$. To see this, consider the function $\widetilde{f}(t)=f(t) / C$ that is bounded by 1 and Lipschitz continuous with constant 1 . Let $\widetilde{\mu}^{*}$ be the linear functional associated to $\widetilde{f}(t)$. Then $\mu^{*}=C \widetilde{\mu}^{*}$ is a linear functional such that $\left\langle\mu^{*}, \delta_{t}\right\rangle=f(t)$ and $\left\|\mu^{*}\right\|_{\mathcal{M}^{\prime}} \leq C$. From the uniqueness of $\widetilde{\mu}^{*}$ we get the uniqueness of $\mu^{*}$.

Now we assume that $C=1$. We define $\mu^{*}$ on the space spanned by $\left(\delta_{t}\right)_{t \in \mathbb{R}_{+}}$(the space $\left.M\right)$ as $\left\langle\mu^{*}, \delta_{t}\right\rangle=f(t)$. We shall prove that $\mu^{*}$ is indeed a continuous linear functional on $M$. Linearity is obvious. Since $f \in \mathcal{D}$, for any $\mu \in M$ we have

$$
\left|\left\langle\mu^{*}, \mu\right\rangle\right|=\left|\sum_{t \in \mathbb{R}_{+}} \mu(t) f(t)\right| \leq \sup _{h \in \mathcal{D}}\left|\sum_{t \in \mathbb{R}_{+}} \mu(t) h(t)\right|=\|\mu\|_{M} .
$$

Hence $\left\|\mu^{*}\right\|_{M^{\prime}} \leq 1$. We can extend $\mu^{*}$ to the whole of $\mathcal{M}$ as a continuous linear functional of norm 1 . This extension is unique since $\mathcal{M}$ is a completion of $M$.

4. Random variables on $\mathcal{M}$. Let $(\Omega, \mathcal{F}, \mathbb{P})$ be a probability space and $X$ a mapping defined on $\Omega$ with values in the Banach space $\mathcal{M}$. Then $X$ is a simple random variable if it is measurable and admits a finite number of values, i.e. there exists $N \in \mathbb{N}$, a sequence $\left(\mu_{n}\right)_{n=1}^{N} \subset \mathcal{M}$ and $N$ disjoint measurable sets $\left(A_{n}\right)_{n=1}^{N}$ such that $A_{n} \in \mathcal{F}, \bigcup_{n=1}^{N} A_{n}=\Omega$ and $X=\sum_{n=1}^{N} \mu_{n} 1_{A_{n}}$. A mapping $X$ is strongly measurable if there exists a sequence of simple random variables converging to $X$ a.s. in the norm of $\mathcal{M}$.

Definition 4.1. The space $L_{\mathbb{P}}^{1}(\Omega, \mathcal{M})$ consists of all strongly measurable random variables $X$ for which

$$
\|X\|_{L_{\mathbb{P}}^{1}(\Omega, \mathcal{M})}=\mathbb{E}\|X\|_{\mathcal{M}}<\infty .
$$

LEMma $4.2([8]) . L_{\mathbb{P}}^{1}(\Omega, \mathcal{M})$ with the norm $\|\cdot\|_{L_{\mathbb{P}}^{1}(\Omega, \mathcal{M})}$ is a Banach space.

Following Schwartz $([7])$ we construct the dual space to $L_{\mathbb{P}}^{1}(\Omega, \mathcal{M})$. A mapping $\Phi: \Omega \rightarrow \mathcal{M}^{\prime}$, where $\mathcal{M}^{\prime}$ is dual to $\mathcal{M}$, is called ${ }^{*}$-weakly measurable if for any $x \in \mathcal{M}$ the function $\omega \mapsto\langle\Phi(\omega), x\rangle$ is measurable. Let 
$\mathcal{L}^{\infty}\left(\Omega, \mathcal{M}^{\prime}\right)$ be the set of all *-weakly measurable mappings for which the function $\Phi \mapsto \inf \left\{K \geq 0:\|\Phi\|_{\mathcal{M}^{\prime}} \leq K\right.$ a.s. $\}$ is finite. We define an equivalence relation in $\mathcal{L}^{\infty}\left(\Omega, \mathcal{M}^{\prime}\right)$ by $\Phi \sim \Psi$ if $\forall x \in \mathcal{M},\langle\Phi, x\rangle=\langle\Psi, x\rangle$ a.s.

Definition 4.3. We define

$$
L_{*}^{\infty}\left(\Omega, \mathcal{M}^{\prime}\right)=\mathcal{L}^{\infty}\left(\Omega, \mathcal{M}^{\prime}\right) / \sim
$$

and

$$
\|\Phi\|_{L_{*}^{\infty}\left(\Omega, \mathcal{M}^{\prime}\right)}=\inf _{\phi \sim \Phi}\|\phi\|_{\mathcal{L}^{\infty}\left(\Omega, \mathcal{M}^{\prime}\right)}=\inf \left\{K \geq 0:\|\Phi\|_{\mathcal{M}^{\prime}} \leq K \text { a.s. }\right\} .
$$

Theorem $4.4([7])$. $L_{*}^{\infty}\left(\Omega, \mathcal{M}^{\prime}\right)$ with the norm $\|\cdot\|_{L_{*}^{\infty}\left(\Omega, \mathcal{M}^{\prime}\right)}$ is a Banach space. Moreover, it is dual to $L_{\mathbb{P}}^{1}(\Omega, \mathcal{M})$. Every $\Psi \in L_{*}^{\infty}\left(\Omega, \mathcal{M}^{\prime}\right)$ defines a linear functional on $L_{\mathbb{P}}^{1}(\Omega, \mathcal{M})$ by

$$
L_{\mathbb{P}}^{1}(\Omega, \mathcal{M}) \ni X \mapsto\langle\Psi, X\rangle_{\left\langle L_{*}^{\infty}\left(\Omega, \mathcal{M}^{\prime}\right), L_{\mathbb{P}}^{1}(\Omega, \mathcal{M})\right\rangle}=\mathbb{E}\langle\Psi, X\rangle_{\left\langle\mathcal{M}^{\prime}, \mathcal{M}\right\rangle} .
$$

As a final result we will prove a simple technical lemma.

LEMma 4.5. Let $\tau$ be a non-negative bounded random variable $(\tau<K$ a.s. for some $K \in \mathbb{R})$ and $Y \in L^{1}(\Omega, \mathcal{F}, \mathbb{P})$. Then $Y \delta_{\tau} \in L_{\mathbb{P}}^{1}(\Omega, \mathcal{M})$.

Proof. We will construct a sequence of simple random variables $X_{n} \in$ $L_{\mathbb{P}}^{1}(\Omega, \mathcal{M})$ with limit $Y \delta_{\tau}$. Let $Y_{n}$ be a sequence of simple random variables converging to $Y$ a.s. Fix $n \in \mathbb{N}$. Set $A_{k}=\{\tau \in[k K / n,(k+1) K / n)\}$ for $k=0, \ldots, n-1$. Put

$$
X_{n}=\sum_{k=0}^{n-1} Y_{n} 1_{A_{k}} \delta_{k K / n} .
$$

Then the pointwise convergence of $X_{n}$ to $Y \delta_{\tau}$ follows from Lemma 3.2.

5. Absence of free lunch and its equivalent characterization. In this Section we will establish a definition of no free lunch for the model presented in Section 2. Moreover, we will derive an analog of the Fundamental Theorem of Asset Pricing - an equivalent characterization of the absence of free lunch. It will be done in the spirit of Jouini and Napp's paper [3].

First, we will find a representation of any investment as an element of the space $L_{\mathbb{P}}^{1}(\Omega, \mathcal{M})$. We denote by $\Lambda$ the subset of $L_{\mathbb{P}}^{1}(\Omega, \mathcal{M})$ of all random variables that can be written in the form

$$
\gamma_{1} \delta_{\tau_{1}}+\ldots+\gamma_{m} \delta_{\tau_{m}}
$$

for some $m \in \mathbb{N}$, a sequence $\tau_{1}, \ldots, \tau_{m}$ of bounded stopping times and $\gamma_{i} \in L^{1}\left(\Omega, \mathcal{F}_{\tau_{i}}, \mathbb{R}\right), i=1, \ldots, m$. The subset of all non-negative elements in $\Lambda$ will be denoted by $\Lambda_{+}$:

$$
\Lambda_{+}=\left\{\gamma \in \Lambda: \gamma=\sum_{i=1}^{m} \gamma_{i} \delta_{\tau_{i}}, \gamma_{i} \geq 0 \text { a.s. }\right\} .
$$


Lemma 5.1. For any $\gamma \in \Lambda$ with representation $\gamma=\sum_{i=1}^{m} \gamma_{i} \delta_{\tau_{i}}$,

$$
\|\gamma\|_{L_{\mathbb{P}}^{1}(\Omega, \mathcal{M})} \leq \sum_{i=1}^{m} \mathbb{E}\left|\gamma_{i}\right| .
$$

Proof. It suffices to prove the lemma for $\gamma$ of the form $\gamma=\gamma_{1} \delta_{\tau_{1}}+\gamma_{2} \delta_{\tau_{2}}$ :

$$
\begin{aligned}
\|\gamma\|_{L_{\mathbb{P}}^{1}(\Omega, \mathcal{M})}=\mathbb{E}\|\gamma\|_{\mathcal{M}} & \leq \mathbb{E}\left(\left|\gamma_{1}\right|\left\|\delta_{\tau_{1}}\right\|_{\mathcal{M}}+\left|\gamma_{2}\right|\left\|\delta_{\tau_{2}}\right\|_{\mathcal{M}}\right) \\
& =\mathbb{E}\left|\gamma_{1}\right|\left\|\delta_{\tau_{1}}\right\|_{\mathcal{M}}+\mathbb{E}\left|\gamma_{2}\right|\left\|\delta_{\tau_{2}}\right\|_{\mathcal{M}}=\mathbb{E}\left|\gamma_{1}\right|+\mathbb{E}\left|\gamma_{2}\right| .
\end{aligned}
$$

Having all necessary tools we establish an isomorphism between $\Lambda$ and the set $\Delta$ of all investments:

$$
\Delta \ni \sum_{i=1}^{m} \phi_{\tau_{i}} 1_{\tau_{i}=t} \leftrightarrow \sum_{i=1}^{m} \phi_{\tau_{i}} \delta_{\tau_{i}} \in \Lambda .
$$

Note that the representations in $\Lambda$ and in $\Delta$ are not unique. From now on we will treat both representations equivalently; an investment is a stochastic process or an element of $L_{\mathbb{P}}^{1}(\Omega, \mathcal{M})$ depending on the context.

In the future considerations we will also need the following notation. Let $\left(h_{t}\right)_{t \in \mathbb{R}_{+}}$be a stochastic process and $\Phi=\sum_{i=1}^{m} \phi_{\tau_{i}} 1_{\tau_{i}=t}$ an element of $\Delta$. Then by $\mathbb{E}\left[\sum_{t} \Phi_{t} h_{t}\right]$ we mean the number $\sum_{i=1}^{m} \mathbb{E}\left[\phi_{\tau_{i}} h_{\tau_{i}}\right]$.

We now come to the notion of absence of arbitrage and free lunch. The first one is proper for simplest models. In the more complicated framework one has to introduce a stronger condition which is usually called "no free lunch". It will be crucial to obtain a reasonable characterization of markets without the possibility of getting free lunch.

Definition 5.2. There is no arbitrage on the market $J$ iff $J \cap \Lambda_{+}=\{0\}$.

Definition 5.3. There is no free lunch on the market $J$ iff $\overline{J-\Lambda_{+}} \cap$ $\Lambda_{+}=\{0\}$, where the closure is taken in the norm of $L_{\mathbb{P}}^{1}(\Omega, \mathcal{M})$.

Let $\left(h_{t}\right)_{t \in \mathbb{R}_{+}}$be a measurable process. By $\left(h_{t}^{o}\right)_{t \in \mathbb{R}_{+}}$we will denote its optional projection.

TheOREM 5.4. Let $J \subset \Lambda$ be a positive convex cone and $\mu$ be a non-null element of $\Lambda$. If $\mu \notin \overline{J-\Lambda_{+}}$(the closure in the norm of $L_{\mathbb{P}}^{1}(\Omega, \mathcal{M})$ ), then there exists a (not necessarily adapted) measurable process $\left(h_{t}\right)_{t \in \mathbb{R}_{+}}$such that

(i) $\mathbb{P}\left(h_{t}^{\mathrm{o}} \geq 0,\left|h_{t}\right| \leq M^{h} \forall t \in \mathbb{R}_{+}\right)=1$ (boundedness),

(ii) $\mathbb{P}\left(\left|h_{t}-h_{s}\right| \leq M^{h}|t-s| \forall t, s \in \mathbb{R}_{+}\right)=1$ (Lipschitz continuity),

(iii) $\mathbb{E}\left[\sum_{t} \mu_{t} h_{t}\right]>1$,

(iv) $\mathbb{E}\left[\sum_{t} \Phi_{t} h_{t}\right] \leq 0$ for every $\Phi \in J$.

The constant $M^{h}$ depends on $\mu$ and $J$.

Proof. Let $C=J-\Lambda_{+}$. We apply the Hahn-Banach separation theorem (see Theorem IV.6.3 in Yosida [8]) in the space $L_{\mathbb{P}}^{1}(\Omega, \mathcal{M})$ to the element 
$\mu$ and the closed convex set $\bar{C}(0 \in C)$. We find a linear functional $\mathfrak{f} \in$ $L_{*}^{\infty}\left(\Omega, \mathcal{M}^{\prime}\right)$ such that $\mathfrak{f}(\mu)>1$ and $\mathfrak{f} \leq 1$ on $C$. We recall the properties of $\mathfrak{f}$ (see Section 4$)$ :

(a) $\langle\mathfrak{f}, \nu\rangle_{\left\langle\mathcal{M}^{\prime}, \mathcal{M}\right\rangle}$ is measurable for any element $\nu \in \mathcal{M}$,

(b) $\|\mathfrak{f}\|_{L_{*}^{\infty}\left(\Omega, \mathcal{M}^{\prime}\right)}=\inf \left\{K \geq 0:\|\mathfrak{f}\|_{\mathcal{M}^{\prime}} \leq K\right.$ a.s. $\}$.

We have $\mathfrak{f} \leq 0$ on $C$ since $C$ is a positive cone.

Let $h_{t}=\left\langle\mathfrak{f}, \delta_{t}\right\rangle_{\left\langle\mathcal{M}^{\prime}, \mathcal{M}\right\rangle}$ for $t \in \mathbb{R}_{+}$. Then by (a), $h_{t}$ is a random variable for all $t \in \mathbb{R}_{+}$. From Lemma 3.4 and (b) we deduce that all trajectories of $\left(h_{t}\right)_{t \in \mathbb{R}_{+}}$are Lipschitz continuous and bounded with constant independent of $\omega \in \Omega$, i.e. $\forall t, s \in \mathbb{R}_{+},\left|h_{t}(\omega)-h_{s}(\omega)\right| \leq M^{h}|t-s|$ and $\left|h_{t}(\omega)\right| \leq M^{h}$ for almost all $\omega \in \Omega$. Then a simple argument shows that $\left(h_{t}\right)_{t \in \mathbb{R}_{+}}$is a measurable process (see for example Remark 1.14 in Karatzas-Shreve [4]).

We claim that for $t \in \mathbb{R}_{+}, h_{t}^{o} \geq 0$ a.s. Assume the contrary. Thus there exists $s \in \mathbb{R}_{+}$such that $\mathbb{P}\left(h_{s}^{\mathrm{o}}<0\right)>0$. Let $V=\left\{h_{s}^{\circ}<0\right\}$ and $L_{\mathbb{P}}^{1}(\Omega, \mathcal{M}) \ni$ $\Psi: \omega \mapsto-1_{V}(\omega) \delta_{s}$. Obviously $\Psi \in-\Lambda_{+}$, so $\Psi \in C$. Hence

$$
\langle\mathfrak{f}, \Psi\rangle_{\left\langle L_{*}^{\infty}\left(\Omega, \mathcal{M}^{\prime}\right), L_{\mathbb{P}}^{1}(\Omega, \mathcal{M})\right\rangle}=\mathbb{E}\left[\langle\mathfrak{f}, \Psi\rangle_{\left\langle\mathcal{M}^{\prime}, \mathcal{M}\right\rangle}\right] \leq 0 .
$$

But

$$
\mathbb{E}\left[\langle\mathfrak{f}, \Psi\rangle_{\left\langle\mathcal{M}^{\prime}, \mathcal{M}\right\rangle}\right]=\mathbb{E}\left[-1_{V}\left\langle\mathfrak{f}, \delta_{s}\right\rangle_{\left\langle\mathcal{M}^{\prime}, \mathcal{M}\right\rangle}\right]=\mathbb{E}\left(-1_{V} h_{s}^{o}\right)>0 .
$$

This is a contradiction. The right continuity of trajectories of $h_{t}^{o}$ (see Theorem VI.7.10 of Rogers-Williams [6]) shows that $\mathbb{P}\left(h_{t}^{\circ} \geq 0 \forall t \in \mathbb{R}_{+}\right)=1$.

For the proof of (iv) we recall the definition

$$
\mathbb{E}\left[\sum_{t} \Phi_{t} h_{t}\right]=\mathbb{E}\langle\mathfrak{f}, \Phi\rangle_{\left(\mathcal{M}^{\prime}, \mathcal{M}\right)}
$$

Now we come to the main part of this section - the analog of the Fundamental Theorem of Asset Pricing. To establish it we will need a technical assumption which is satisfied by most of the models. It assures that we can always find an investment that enables us to transfer some money from any moment to one of the specified moments in the future with positive probability. It is a kind of roulette or lottery condition.

DeFinition 5.5. A market $J$ satisfies the roulette condition if there exists a sequence $\left(\sigma_{n}\right)_{n \in \mathbb{N}}$ of bounded stopping times such that for each bounded stopping time $\tau$ and each subset $A \in \mathcal{F}_{\tau}$ with positive probability we can find an investment $\Phi$ in $J$ such that

- $\mathbb{P}\left(\Phi_{s}=0 \forall s<\tau\right)=1$,

- $\Phi_{\tau}=0$ a.s. on $A^{\mathrm{c}}$,

- $\mathbb{P}\left(\Phi_{s} \geq 0 \forall s>\tau\right)=1$,

- there exists $n \in \mathbb{N}$ with $\mathbb{P}\left(\Phi_{\sigma_{n}}>0\right)>0$. 
THEOREM 5.6. Assume that the market $J$ satisfies the roulette condition. There is no free lunch in $J$ iff there exists a measurable process $\left(g_{t}\right)_{t \in \mathbb{R}_{+}}$such that

(i) $\mathbb{P}\left(\left|g_{t}\right| \leq M^{g} \forall t \in \mathbb{R}_{+}\right)=1$ (boundedness),

(ii) $\mathbb{P}\left(\left|g_{t}-g_{s}\right| \leq M^{g}|t-s| \forall t, s \in \mathbb{R}_{+}\right)=1$ (Lipschitz continuity),

(iii) $\mathbb{E}\left[g_{\tau} \mid \mathcal{F}_{\tau}\right]>0$ a.s. for any bounded stopping time $\tau$,

(iv) $\mathbb{E}\left[\sum_{t} \Phi_{t} g_{t}\right] \leq 0$ for $\Phi \in J$.

Every process $g_{t}$ satisfying the above conditions is called a discount process for $J$.

Proof. Assume that there exists a discount process $g_{t}$ for $J$. We have to show that $\overline{J-\Lambda_{+}} \cap \Lambda_{+}=\{0\}$. Let $X^{n} \in J-\Lambda_{+}$be a sequence converging to $X \in \Lambda_{+}$in the norm of $L_{\mathbb{P}}^{1}(\Omega, \mathcal{M})$.

First we construct a linear functional $\Psi$ such that $\Psi(\Phi)=\mathbb{E} \sum_{t} \Phi_{t} g_{t}$ for any $\Phi \in \Lambda$. Let $H$ be the linear subspace of $L_{\mathbb{P}}^{1}(\Omega, \mathcal{M})$ spanned by the random variables $1_{A} \delta_{t}$ for $A \in \mathcal{F}_{t}, t \in \mathbb{R}_{+}$. On $H$ we set $\Psi\left(1_{A} \delta_{t}\right)=\mathbb{E} 1_{A} g_{t}$. Linearity of this function is clear. We only have to show that $\Psi$ is continuous. Let $Y \in H$. We can write $Y=\sum_{k=1}^{K} \alpha_{k} 1_{A_{k}} \delta_{t_{k}}$ for some $K, A_{k} \in \mathcal{F}_{t_{k}}$, $t_{k} \in \mathbb{R}_{+}, \alpha_{k} \in \mathbb{R}, k=1, \ldots, K$. Hence

$$
\begin{aligned}
\Psi(Y) & =\sum_{k=1}^{K} \alpha_{k} \Psi\left(1_{A_{k}} \delta_{t_{k}}\right)=\sum_{k=1}^{K} \alpha_{k} \mathbb{E}\left[1_{A_{k}} g_{t_{k}}\right] \\
& =\mathbb{E} \sum_{k=1}^{K} \alpha_{k} 1_{A_{k}} g_{t_{k}}=\int \sum_{\Omega}^{K} \alpha_{k=1} 1_{A_{k}}(\omega) g_{t_{k}}(\omega) d \mathbb{P}(\omega) .
\end{aligned}
$$

For almost all $\omega \in \Omega$, the function $t \mapsto g_{t}(\omega)$ is Lipschitz continuous with constant $M^{g}$ and is bounded by $M^{g}$. Fix $\omega \in \Omega$. Then by Lemma 3.5, $g_{t}(\omega)$ defines a continuous linear functional on $\mathcal{M}$ with norm bounded by $M^{g}$. Since $\sum_{k=1}^{K} \alpha_{k} 1_{A_{k}}(\omega) \delta_{t_{k}} \in \mathcal{M}$ we obtain

$$
\left|\sum_{k=1}^{K} \alpha_{k} 1_{A_{k}}(\omega) g_{t_{k}}(\omega)\right| \leq M^{g}\left\|\sum_{k=1}^{K} \alpha_{k} 1_{A_{k}}(\omega) \delta_{t_{k}}\right\|_{\mathcal{M}}=M^{g}\|Y(\omega)\|_{\mathcal{M}} .
$$

Thus

$$
\begin{aligned}
|\Psi(Y)| & =\left|\int_{\Omega} \sum_{k=1}^{K} \alpha_{k} 1_{A_{k}}(\omega) g_{t_{k}}(\omega) d \mathbb{P}(\omega)\right| \leq \int_{\Omega}\left|\sum_{k=1}^{K} \alpha_{k} 1_{A_{k}}(\omega) g_{t_{k}}(\omega)\right| d \mathbb{P}(\omega) \\
& \leq \int_{\Omega} M^{g}\|Y(\omega)\|_{\mathcal{M}} d \mathbb{P}(\omega)=M^{g}\|Y\|_{L_{\mathbb{P}}^{1}(\Omega, \mathcal{M})} .
\end{aligned}
$$

We extend $\Psi$ to the whole of $L_{\mathbb{P}}^{1}(\Omega, \mathcal{M})$ as a continuous linear functional (see Yosida [8, IV.5.1]). Observe that $\Psi\left(1_{A} \delta_{\tau}\right)=\mathbb{E} 1_{A} g_{\tau}$ for any bounded 
stopping time $\tau$ and $A \in \mathcal{F}_{\tau}$. Let $\tau_{n}$ be a sequence of stopping times admitting a finite number of values and converging to $\tau$ almost surely. By Lemma $3.2,1_{A} \delta_{\tau_{n}} \stackrel{\mathcal{M}}{\longrightarrow} 1_{A} \delta_{\tau}$ a.s. and from the dominated convergence theorem $1_{A} \delta_{\tau_{n}} \rightarrow 1_{A} \delta_{\tau}$ in $L_{\mathbb{P}}^{1}(\Omega, \mathcal{M})$. Thus $\Psi\left(1_{A} \delta_{\tau_{n}}\right) \rightarrow \Psi\left(1_{A} \delta_{\tau}\right)$. On the other hand, $\mathbb{E} 1_{A} g_{\tau_{n}} \rightarrow \mathbb{E} 1_{A} g_{\tau}$ by the dominated convergence theorem ( $g_{t}$ is bounded by $\left.M^{g}\right)$. Then $\Psi\left(1_{A} \delta_{\tau}\right)=\mathbb{E} 1_{A} g_{\tau}$. A similar argument shows that $\Psi\left(\Theta \delta_{\tau}\right)=\mathbb{E} \Theta g_{\tau}$ for any $\Theta \in L^{1}\left(\Omega, \mathcal{F}_{\tau}, \mathbb{P}\right)$.

Hence it is clear that $\Psi\left(X^{n}\right)=\mathbb{E}\left[\sum_{t} X_{t}^{n} g_{t}\right]$ and $\Psi(X)=\mathbb{E}\left[\sum_{t} X_{t} g_{t}\right]$. By continuity of $\Psi$ we obtain $\Psi\left(X^{n}\right) \rightarrow \Psi(X)$. Therefore $\Psi(X) \leq 0$ since $\Psi\left(X^{n}\right) \leq 0$. However, $X \in \Lambda_{+}$, so $\Psi(X) \geq 0$ (from the condition $\mathbb{E}\left[g_{\tau} \mid \mathcal{F}_{\tau}\right]$ $>0$ a.s. for any bounded stopping time $\tau$ ). This implies that $X=0$.

Suppose now that there is no free lunch in $J$. Let $\mathcal{G}$ be the set of equivalence classes of measurable processes $g_{t}$ with $\mathbb{P}\left(\left|g_{t}-g_{s}\right| \leq M^{g}|t-s|, g_{t}^{\mathrm{o}} \geq 0\right.$, $\left.\left|g_{t}\right| \leq M^{g} \forall t, s \in \mathbb{R}_{+}\right)=1$ and $\mathbb{E} \sum_{t} \Phi_{t} g_{t} \leq 0$ for $\Phi \in J$. Notice that the null process is contained in $\mathcal{G}$.

We will show that for any bounded stopping time $\tau$ there exists a process $g^{\tau} \in \mathcal{G}$ such that $\mathbb{E}\left[g_{\tau}^{\tau} \mid \mathcal{F}_{\tau}\right]>0$ a.s. Let $\mathcal{H}$ be the following family of equivalence classes of subsets of $\Omega$ :

$$
\mathcal{H}=\left\{A \in \mathcal{F}: \exists h \in \mathcal{G}\left\{\mathbb{E}\left[h_{\tau} \mid \mathcal{F}_{\tau}\right] \neq 0\right\}=A \text { a.s. }\right\} .
$$

We claim that there exists a set of positive measure in $\mathcal{H}$. Fix $\mu=\delta_{\tau} \in$ $\Lambda_{+} \backslash\{0\}$. We apply Theorem 5.4 with $\mu=\delta_{\tau}$ to obtain a process $h \in \mathcal{G}$ such that $\mathbb{E} h_{\tau}>1$. Hence the set $\left\{\mathbb{E}\left[h_{\tau} \mid \mathcal{F}_{\tau}\right] \neq 0\right\}$ must have a positive measure. A simple argument shows that the family $\mathcal{H}$ is closed under countable sums. Let $A_{n} \in \mathcal{H}$ be a sequence of sets. Let $g^{n}$ be a process for which $A_{n}=$ $\left\{\mathbb{E}\left[g_{\tau}^{n} \mid \mathcal{F}_{\tau}\right] \neq 0\right\}$. Put $h=\sum_{n=1}^{\infty}\left(2^{n} M^{g^{n}}\right)^{-1} g^{n}$. Then $h \in \mathcal{G}$ and $\left\{\mathbb{E}\left[h_{\tau} \mid \mathcal{F}_{\tau}\right]\right.$ $\neq 0\}=\bigcup A_{n}$ a.s. Hence there exists $S^{*} \in \mathcal{H}$ such that $\mathbb{P}\left(S^{*}\right)=\sup \{\mathbb{P}(S)$ : $S \in \mathcal{H}\}$. If $\mathbb{P}\left(S^{*}\right)<1$ then by Theorem 5.4 applied to $\mu=1_{\Omega \backslash S^{*}} \delta_{\tau}$ we obtain a process $h^{\prime} \in \mathcal{G}$ with $\mathbb{E} 1_{\Omega \backslash S^{*}} h_{\tau}^{\prime}>1$. Considering the process $h+h^{\prime} \in \mathcal{G}$ yields a contradiction.

Using the above results we can find a process $g \in \mathcal{G}$ such that $\mathbb{E}\left[g_{\sigma_{n}} \mid \mathcal{F}_{\sigma_{n}}\right]$ $>0$ a.s. for every $n \in \mathbb{N}$ and

$$
g=\sum_{i=1}^{\infty} \frac{1}{2^{i} M^{g^{\sigma}}} g^{\sigma_{n}} .
$$

We now prove that $\mathbb{E}\left[g_{\tau} \mid \mathcal{F}_{\tau}\right]>0$ a.s. for any bounded stopping time $\tau$. Assume that this is not true, so there exists $\tau$ such that $\mathbb{P}\left(\mathbb{E}\left[g_{\tau} \mid \mathcal{F}_{\tau}\right]=0\right)$ $>0$. Let $B=\left\{\mathbb{E}\left[g_{\tau} \mid \mathcal{F}_{\tau}\right]=0\right\}$. By the roulette condition we can find an investment $\Phi \in J$ null before $\tau$, non-negative after $\tau$, null on $B^{\mathrm{c}}$ and such that $\mathbb{P}\left(\Phi_{\sigma_{n}}>0\right)>0$ for some $n$. We have $\Phi=\sum_{i=1}^{m} \Phi_{\tau_{i}} 1_{\tau_{i}}+\Phi_{\tau} 1_{\tau}+\Phi_{\sigma_{n}} 1_{\sigma_{n}}$ for some $m$ and $\Phi_{\tau_{i}} \geq 0$ a.s. $i=1, \ldots, m$. Then 


$$
\begin{aligned}
\mathbb{E}\left[\sum_{t} \Phi_{t} g_{t}\right] & =\mathbb{E}\left[\sum_{i=1}^{m} \Phi_{\tau_{i}} g_{\tau_{i}}+\Phi_{\tau} g_{\tau}+\Phi_{\sigma_{n}} g_{\sigma_{n}}\right] \\
& =\mathbb{E}\left[\sum_{i=1}^{m} \Phi_{\tau_{i}} g_{\tau_{i}}+\Phi_{\tau} \mathbb{E}\left[g_{\tau} \mid \mathcal{F}_{\tau}\right]+\Phi_{\sigma_{n}} g_{\sigma_{n}}\right] \geq \mathbb{E} g_{\sigma_{n}} \Phi_{\sigma_{n}}>0
\end{aligned}
$$

since $\Phi_{\tau}$ is $\mathcal{F}_{\tau}$-measurable by definition. This yields a contradiction.

REMARK. Notice that the roulette condition was required only to obtain the "only if" part, i.e. to construct a discount process for the market $J$.

Discount processes do not have to be adapted. A mild discount process (Lipschitz continuous) must incorporate the knowledge of the future (one can construct a simple example). However, from the economic point of view, discount factors should be known at each moment, thus they should be adapted. If we ease our requirements for mildness of the discount process we can construct a continuous and adapted discount process.

Corollary 5.7. Assume that the market $J$ satisfies the roulette condition. If there is no free lunch in $J$ then there exists an RCLL adapted process $g_{t}$ such that $\mathbb{E}\left[\sum_{t} \Phi_{t} g_{t}\right] \leq 0$ for $\Phi \in J$ and $\mathbb{P}\left(0<g_{t} \leq M^{g} \forall t \in \mathbb{R}_{+}\right)=1$. If, in addition, the filtration is quasi-left continuous $\left(\mathcal{F}_{\tau-}=\mathcal{F}_{\tau}\right.$ for any previsible stopping time $\tau$ ), then there exists a continuous process $g_{t}$ with the above properties.

Proof. Let $f_{t}$ be a discount process for the market $J$ obtained in Theorem 5.6.

We define $g_{t}$ as an optional projection of $f_{t}$. Notice that $g_{t}$ satisfies condition (iv) of Theorem 5.6. By Theorem VI.7.10 in Rogers-Williams [6], $g_{t}$ has RCLL trajectories. Since $g_{t}=\mathbb{E}\left[f_{t} \mid \mathcal{F}_{t}\right]$ we obtain $0<g_{t} \leq M^{g}$ a.s. for $t \in \mathbb{R}_{+}$. By the right continuity of the trajectories of $g_{t}$ we have $\mathbb{P}\left(0 \leq g_{t} \leq M^{g} \forall t \in \mathbb{R}_{+}\right)=1$.

We only have to prove that $\mathbb{P}\left(0<g_{t} \forall t \in \mathbb{R}_{+}\right)=1$. Let $\gamma_{n}=\inf \{t \in$ $\left.[0, n]: g_{t}=0\right\} \wedge n$ (with the convention $\inf \emptyset=\infty$ ). The random variables $\gamma_{n}$ are stopping times. Then $g_{\gamma_{n}}=\mathbb{E}\left[f_{\gamma_{n}} \mid \mathcal{F}_{\gamma_{n}}\right]>0$ a.s., since $g_{t}$ is an optional projection and $f_{t}$ is a discount process. So $\mathbb{P}\left(\exists t \in[0, n]: g_{t}=0\right)=0$. Consequently, $\mathbb{P}\left(\exists t \in \mathbb{R}_{+}: g_{t}=0\right)=0$, which completes the proof.

Assume now that the filtration is quasi-left continuous. Let $z_{t}$ be a previsible projection of $f_{t}$. Then $z_{t}$ is LCRL (left continuous with right limits). Observe that for any previsible stopping time $\tau$,

$$
g_{\tau} 1_{\tau<\infty}=\mathbb{E}\left[f_{\tau} 1_{\tau<\infty} \mid \mathcal{F}_{\tau}\right]=\mathbb{E}\left[f_{\tau} 1_{\tau<\infty} \mid \mathcal{F}_{\tau-}\right]
$$

since the filtration $\mathcal{F}_{t}$ is quasi-left continuous. Then $g_{t}$ is also a previsible projection. So it is indistinguishable from $z_{t}$. Thus $g_{t}$ is continuous (it is indistinguishable from a continuous process). 
6. Pricing and hedging. In this section we take up a problem of option pricing and hedging. We shall place ourselves in the context of the model of Section 2. The market is described by a positive convex cone $J$ of investment opportunities. Each investment is an adapted process. We assume that the market satisfies the roulette condition and there is no free lunch on it. Thus we are given a set $\mathcal{G}_{J}$ of discount processes for $J$, i.e. for any $g \in \mathcal{G}_{J}$,

- $\mathbb{P}\left(\left|g_{t}\right| \leq M^{g} \forall t \in \mathbb{R}_{+}\right)=1$,

- $\mathbb{P}\left(\left|g_{t}-g_{s}\right| \leq M^{g}|t-s| \forall t, s \in \mathbb{R}_{+}\right)=1$,

- $\mathbb{E}\left[g_{\tau} \mid \mathcal{F}_{\tau}\right]>0$ a.s. for any bounded stopping time $\tau$,

- $\mathbb{E}\left[\sum_{t} \Phi_{t} g_{t}\right] \leq 0$ for $\Phi \in J$.

Throughout this section we will use the following definition of contingent claim:

Definition 6.1. A contingent claim is a pair $(\tau, Y)$, where $\tau$ is a bounded stopping time and $Y$ is a non-negative random variable in $L^{1}\left(\Omega, \mathcal{F}_{\tau}, \mathbb{R}_{+}\right)$.

It is a slight generalization of a widely used notion. We allow a claim to be executed at a random moment. From further results it will be clear that we can also consider contingent claims of the form of investments with non-negative cash flows, i.e. finite sums of contingent claims from Definition 6.1. This enables us to model, apart from classical European options, corporate bonds and more complicated contracts. However, we cannot deal with American options.

There are plenty of methods for determining a price of the contingent claim. We will concentrate on two of them:

- which prices of the option do not lead to arbitrage?

- what is the minimal price that enables hedging of the option?

Arbitrage pricing. A very natural approach to option pricing is based on the notion of no free lunch. A fair price is a price that does not generate free lunch. No one, neither buyer nor seller, gets profit without risk. As we will see later, the set of such prices is an interval, a common result in financial mathematics.

To consider options we have to define an investment representing buying and selling of the option. Let

$$
\Psi_{t}^{(\xi, C, \tau, Y)}=\xi\left(C 1_{t=0}-Y 1_{t=\tau}\right),
$$

where $\xi, C \in \mathbb{R}$. Then $\Psi^{(-1, C, \tau, Y)}$ denotes the investment consisting of buying the option $(\tau, Y)$ at time 0 for the price $C$. A claim $Y$ is paid back at the random time $\tau$. In a natural way, selling is opposite to buying, thus it is represented by $\Psi^{(1, C, \tau, Y)}$.

Definition 6.2. An option price $C$ is called fair if the market $J^{\text {opc }}$ generated by $J$ and the investments $\Psi^{( \pm 1, C, \tau, Y)}$ does not admit free lunch. 
Hence, the set of discount processes $\mathcal{G}_{J \text { opc }}$ is not empty. It is clear that $\mathcal{G}_{\text {Jopc }} \subset \mathcal{G}_{J}$. Let $g \in \mathcal{G}_{J^{\text {opc }}}$. The inequality

$$
\mathbb{E} \sum_{t \in \mathbb{R}_{+}} \Psi_{t}^{( \pm 1, C, \tau, Y)}= \pm\left(C \mathbb{E} g_{0}-\mathbb{E}\left[g_{\tau} Y\right]\right) \leq 0
$$

results in $C \mathbb{E} g_{0}-\mathbb{E}\left[g_{\tau} Y\right]=0$. Thus $C$ is a fair price if and only if there exists $g \in \mathcal{G}_{J}$ such that $C=\mathbb{E}\left[g_{\tau} Y\right] / \mathbb{E} g_{0}$.

Lemma 6.3. Let $C_{1} \leq C_{2}$ be fair prices of the contingent claim $(\tau, Y)$. Then any price from the interval $\left[C_{1}, C_{2}\right]$ is fair.

Proof. Let $g^{1}, g^{2}$ be discount processes for the prices $C_{1}, C_{2}$. Take any $C \in\left[C_{1}, C_{2}\right]$. We can find $a \in[0,1]$ such that $C=a C_{1}+(1-a) C_{2}$. Define

$$
g_{t}=a \frac{g_{t}^{1}}{\mathbb{E} g_{0}^{1}}+(1-a) \frac{g_{t}^{2}}{\mathbb{E} g_{0}^{2}} .
$$

Then $C=\mathbb{E}\left[g_{\tau} Y\right] / \mathbb{E} g_{0}$. It is clear that $g \in \mathcal{G}_{J}$.

The above lemma yields a remarkable characterization of the interval of fair prices. However, it is unspecific about the ends of the interval. We do not know if these prices lead to arbitrage or not.

Corollary 6.4. Let

$$
C^{h}=\sup _{g \in \mathcal{G}_{J}} \frac{\mathbb{E}\left[g_{\tau} Y\right]}{\mathbb{E} g_{0}}, \quad C^{l}=\inf _{g \in \mathcal{G}_{J}} \frac{\mathbb{E}\left[g_{\tau} Y\right]}{\mathbb{E} g_{0}} .
$$

Any price from the open interval $\left(C^{l}, C^{h}\right)$ is fair. Moreover, any price outside the closed interval $\left[C^{l}, C^{h}\right]$ leads to free lunch.

Proof. For any $C \in\left(C^{l}, C^{h}\right)$ we can find fair prices $C_{1} \leq C_{2}$ such that $C \in\left[C_{1}, C_{2}\right]$. By Lemma $6.3, C$ is a fair price. The second assertion is obvious.

Hedging. An agent selling the option wants to know if it is possible to hedge it and what is the minimal amount of money that would enable hedging. Does it have anything to do with fair prices? To address these questions we have to specify what is meant by hedging a contingent claim. At time zero we obtain a certain amount of money. Then we invest the money (we subscribe to investment opportunities available on the market) to get a cash flow at time $\tau$ that covers our obligation $Y$. This intuitive notion is specified in the following definition. We denote by $\Psi^{C}$ the investment $\Psi^{(-1, C, \tau, Y)}$, buying the option.

Definition 6.5. A price $C$ is a hedging price for $(\tau, Y)$ if $\Psi^{C} \in \overline{J-\Lambda_{+}}$. A minimal hedging price is denoted by $C^{s}$ and called the seller price.

If $C$ is a hedging price, then there exists a sequence of investments that in the limit gives $\Psi^{C}$ and some positive cash flows, an available consumption. 
It is still an open question if a seller price exists. Let

$$
h=\inf \{b \geq 0: b \text { is a hedging price }\} .
$$

We will show that $h$ is in fact a minimal hedging price. Obviously if $C$ is a hedging price then $C \geq h$. We prove that $h$ is a hedging price. Let $b_{n}$ be a sequence of hedging prices converging to $h$. Then $\Psi^{b_{n}}$ is a Cauchy sequence in $L_{\mathbb{P}}^{1}(\Omega, \mathcal{M})$ with limit $\Psi^{h}$. From $\Psi^{b_{n}} \in \overline{J-\Lambda_{+}}$we obtain $\Psi^{h} \in \overline{J-\Lambda_{+}}$.

TheOREM 6.6. Let $C^{s}$ be a minimal hedging price and $C^{h}$ the upper limit of the fair price interval from Corollary 6.4. Then $C^{s}=C^{h}$.

Proof. First we will prove $C^{s} \geq C^{h}$. We have shown that $\Psi^{C^{s}} \in \overline{J-\Lambda_{+}}$. Thus for all discount processes $g \in \mathcal{G}_{J}$ we have $\mathbb{E}\left[Y g_{\tau}\right]-C^{s} \mathbb{E} g_{0} \leq 0$. Hence

$$
C^{s} \geq \frac{\mathbb{E}\left[Y g_{\tau}\right]}{\mathbb{E} g_{0}} \quad \text { for every } g \in \mathcal{G}_{J}
$$

and finally

$$
C^{s} \geq \sup _{g \in \mathcal{G}_{J}} \frac{\mathbb{E}\left[Y g_{\tau}\right]}{\mathbb{E} g_{0}}=C^{h} .
$$

If $C^{h}=\infty$ then $C^{s}=\infty$. Let $C^{h}<\infty$. We will show that $C^{h}$ is a hedging price. Assume otherwise. Thus $\Psi^{C^{h}} \notin \overline{J-\Lambda_{+}}$. By Theorem 5.4 there exists a process $h$ satisfying conditions (i)-(iii) for a discount process (see Theorem 5.6) and such that

$$
\mathbb{E}\left[Y h_{\tau}\right]-C^{h} \mathbb{E} h_{0}>1 .
$$

The definition of $C^{h}$ grants the existence of a discount processes $g \in \mathcal{G}_{J}$ such that

$$
C^{h}-\frac{1}{2}=\frac{\mathbb{E}\left[Y g_{\tau}\right]}{\mathbb{E} g_{0}}
$$

Let $\widetilde{g}=g / \mathbb{E} g_{0}+h$. We can easily check that $\widetilde{g}$ satisfies conditions (i), (ii), (iv) for a discount process since both $h$ and $g$ do. Condition (iii) results from the fact that $\mathbb{E}\left[h_{\tau} \mid \mathcal{F}_{\tau}\right] \geq 0$ a.s. and $\mathbb{E}\left[g_{\tau} \mid \mathcal{F}_{\tau}\right]>0$ a.s. for any bounded stopping time $\tau$. Thus $\widetilde{g} \in \mathcal{G}_{J}$ and

$$
C^{h} \geq \frac{\mathbb{E}\left[Y \widetilde{g}_{\tau}\right]}{\mathbb{E} \widetilde{g}_{0}}
$$

On the other hand, from (2) we obtain

$$
\begin{aligned}
C^{h} \mathbb{E} \widetilde{g}_{0} & =C^{h}+C^{h} \mathbb{E} h_{0}=\left(C^{h}-\frac{1}{2}\right)+\frac{1}{2}+C^{h} \mathbb{E} h_{0} \\
& =\frac{\mathbb{E}\left[Y g_{\tau}\right]}{\mathbb{E} g_{0}}+\frac{1}{2}+C^{h} \mathbb{E} h_{0}<\frac{\mathbb{E}\left[Y g_{\tau}\right]}{\mathbb{E} g_{0}}+\frac{1}{2}+\mathbb{E}\left[Y h_{\tau}\right]-1 \\
& =\mathbb{E}\left[Y \widetilde{g}_{\tau}\right]+\frac{1}{2}-1<\mathbb{E}\left[Y \widetilde{g}_{\tau}\right]-\frac{1}{2} .
\end{aligned}
$$


Combining the last two results yields a contradiction:

$$
\mathbb{E}\left[Y \widetilde{g}_{\tau}\right] \leq C^{h} \mathbb{E} \widetilde{g}_{0}<\mathbb{E}\left[Y \widetilde{g}_{\tau}\right]-\frac{1}{2}
$$

\section{References}

[1] N. H. Bingham and R. Kiesel, Risk-Neutral Valuation. Pricing and Hedging of Financial Derivatives, Springer, 1998.

[2] M. Harrison and D. Kreps, Martingales and arbitrage in multiperiod security markets, J. Econom. Theory 20 (1979), 381-408.

[3] E. Jouini and C. Napp, Arbitrage and investment opportunities, Finance Stoch. 5 (2001), 305-325.

[4] I. Karatzas and S. E. Shreve, Brownian Motion and Stochastic Calculus, Springer, 1991.

[5] C. Napp, Pricing issues with investment flows. Applications to market models with frictions, J. Math. Econom. 35 (2001), 383-408.

[6] L. C. G. Rogers and D. Williams, Diffusions, Markov Processes and Martingales, Cambridge Univ. Press, 2000.

[7] L. Schwartz, Fonctions mesurables et ${ }^{*}$-scalairement mesurables, mesures banachiques majorées, martingales banachiques, et propriété de Radon-Nikodym, exposés 4, 5 et 6 , Séminaire Maurey-Schwartz, École Polytechnique, 1974-1975.

[8] K. Yosida, Functional Analysis, Springer, 1966.

Institute of Mathematics

Polish Academy of Sciences

P.O. Box 21

00-956 Warszawa, Poland

E-mail: j.palczewski@impan.gov.pl

Received on 1.10.2002;

revised version on 10.4.2003 